\title{
Computerised Telephone Billing System
}

\author{
Allison Chukwuma Romanus \\ (Education Secretariat, FCT Universal Basic Education Board Area 2 Section 1P.M.B 163 Garki, Abuja \\ Nigeria)
}

\begin{abstract}
The need for effective and efficient telephone billing system in Nigerian telecommunication industries is increasing everyday as a result of the match towards technological advancement. As such, this study tried to investigate the existing telephone billing system used in the various telecommunication industries in Nigeria with a view to improving on them. To accomplish this, the researcher developed comparative analysis for five telecommunication industries in Nigeria. Etisalat Network was chosen as a case study because it is the network that has the best quality of service and has lowest customer tariff with an effective and responsive customer care unit. Interestingly, the billing system of Etisalat network was discovered to have some shortcomings such as customer name, town name and town code. All these parameters are not displayed in the bill after calls. This research has therefore made an attempt to incorporate the three parameters which did not exist in the Etisalat call summary receipt. This task was achieved using Software Engineering Approach and the Program Source Code was developed using Turbo Pascal version 6.0.
\end{abstract}

Keywords: Billing, Customer friendly, Effective call summary, Parameter, Service quality.

\section{Introduction}

Organizations and in fact the general public needs information for day to day running of their affairs, planning and control or for the reformation of their polices or strategies.

The telephone billing system is mathematical, statistical and logical processes carried out on figures or data obtained from concern telecommunication customers with a view to producing information about billing status due for each customer which can be displayed as a summary report. The purpose of this paper therefore, is to introduce some new parameters in the call summary receipt of telecom operators. The program thus calculates the telephone bills automatically. It does almost every work which is related to automatic telephone billing connection system via- new connection, customer record modification viewing customer records and all works related to rate of bills, meter reading in addition to bill calculation and bill generation. "Telephone Billing System" is developed as per seeing the increasing requirement to speed up the work and incorporate a new work culture. Thus new software has been proposed aim at improving work efficiency, saving time and to provide greater flexibility and customer friendly billing system.

The telephone billing system therefore is a combination of customers' data and telecommunications' components to carry out billing routines described above.

The billing department of most telecommunication organizations also performs the following under listed routines

(a) Gathering time information on (incoming outgoing calls)

(b) Gathering other necessary data such as customer name, location, zone, etc to compute the customers' bill

(c) Adding some attractive incentives such as $5 \%$ or $2 \%$ discount for every customer and above that brought N1000, recharge card.

(d) Reducing tariff for old customers

(e) Making necessary reduction/increment as at when due such as peak hour and off peak hours

(f) Building in all the necessary tax in customers recharge card like VAT and others

(g) Keeping the billing statistics for reference purposes

(h) Overall maintenance of required file

The billing system could be designed to cater for the needs of government agencies, private companies, public institutes

The legal system has a significant influence on the design of telephone billing procedures for example; certain passed laws state other procedures that should be carried out alongside billing calculation. The law has a government agency called Nigerian Communication Commission (NCC) which has the sole right to monitor and regulate the telecommunications industries in Nigeria.[2][3] 


\section{Historical background}

The idea of Cell based mobile radio system was first conceived at Bell Laboratories in (USA) in the early 1970s [1] However, mobile cellular systems were not introduced for commercial use until 1980s during this 1980s; analog cellular telephone systems experienced a very rapid growth in Europe particularly in Scandinavia and in United Kingdom. But in the beginning of cellular system, each country developed its own system which was undesirable situation for the following reasons;

* The equipment was limited to operate only within the boundaries of each country.

* The market for each mobile equipment was limited.

In order to overcome these problems, the Conference of European Posts and Telecommunication (CEPT) in 1982 formed the Special Mobile (GSM) in order to develop a pan - European mobile cellular radio system (with this, GSM acronym because global system for mobile communication). This group came up with a digital system that has to meet the following criteria.

* Spectrum efficiency

* Good subjective voice quality

* International roaming

* Compatibility with other systems such as ISDN

* Low mobile and base stations cost

A digital was adopted by the group (CEPT) as opposed to the then analog cellular systems like AMPs in the United States and TACs in the United Kingdom. The reason for this is on the aspect of quality of service. For example, analog system do pass physical disturbances do decrease signal quality because they produce effects such as fadeouts, crosstalk, hisses etc. On the other hand, digital systems avoid these effects. The phase I of the GSM specifications was published in 1990. Commercial services started mid 1991 and by 1993 there were 36 GSM networks in 22 countries. By the beginning of 1994, there were 1.3 million subscribers worldwide, which grew to 55 million by October 1997 [2][3]

In this paper also, the researcher tries to review a lot of literary works carried out by some other authors and organizations on telephone billing system. The chapter also reviews the activities of the Etisalat Nigeria Plc one the leading telecommunication company as a case study. The Etisalat Nigeria Plc is one of the key player in the telecommunication subsector of the economy. The company started full fledged operation in October 2008[2]. Today it is one of the best telecommunication companies in Nigeria with branches in almost all over the nation. The company has continued to grow from strength to strength with a number of departments and sections [4].

Quelch and Hoff [5], consumer response to product quality also changes dynamically as experience builds up, information accumulates and the cost of quality changes. Omotayo \& Joachim [6], a lot of literatures have named the benefits of retention to an organization. For instance, for an organization such as GSM operator to able to retain a customer, they must have a lot of attractive packages that can attracts customers such as low tariff, good network services, wide coverage etc. Adegoke and Babalola[7], quality of services is an important key performance indicator that is used in determining the efficiency of an industry in terms of service rendered in telecommunication system, accessibility, retainability and connection (voice) quality and three major factors used in evaluating quality of service of an operator. A part from quality, this paper fails to mention about the infrastructure of telecommunication which is one of the major parameter of telecommunication industries.

\section{Conceptual Model}

Fig. I present the conceptual model adopted for the study. The model was adopted from Etisalat Billing System Architecture. The conceptual model has three new parameters namely; customer name, town name and town code as against the existing model used by Etisalat which has only call duration and amount. 


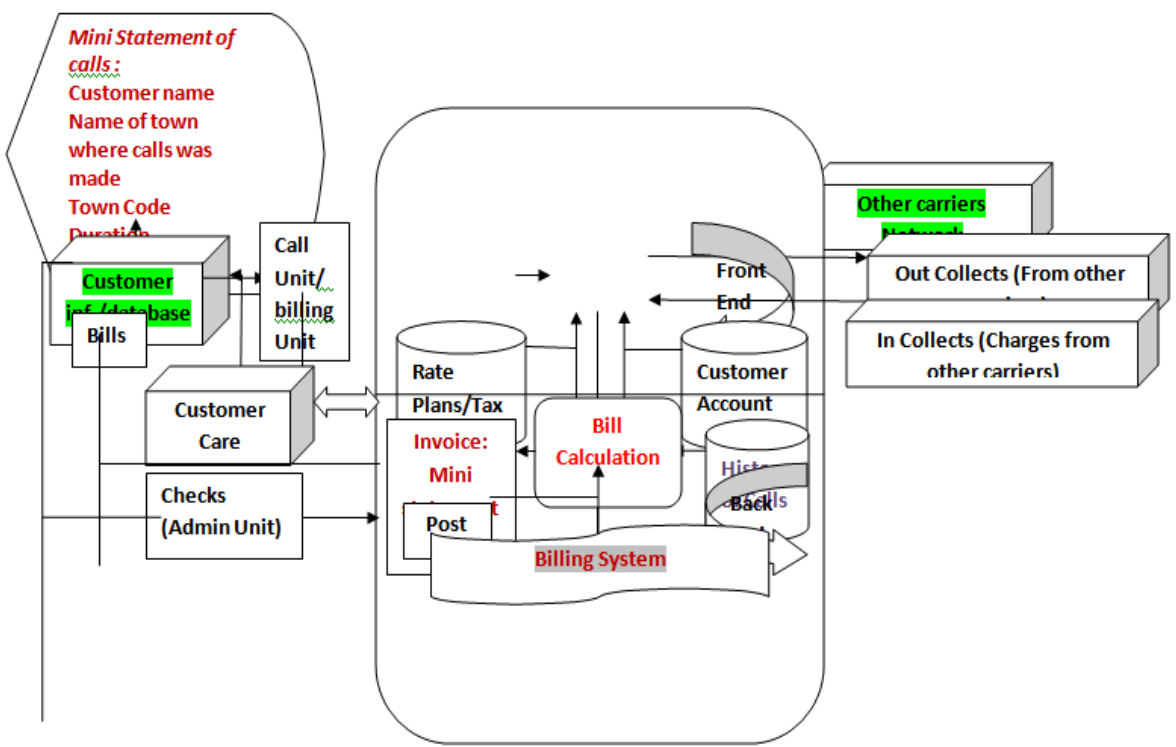

Fig. I Conceptual Model of Billing System

Fig. I has network centre where calls goes out from the network or comes to the network. The usage information goes into the main billing system that has two compartments the front and the back end. The front end contains the rating engine of the billing system. This chamber has three components namely:

Discount Information that handles any bills concerning discounts charges. Rate Plans/Tax Information is aspect of the front that handles any data bundles and tax issues. The Customer Accounts is where the billing engine gets account details of customer. Also the billing engine has the back end which works simultaneously with front end. Calculation of bills using calls history of customer posted to it from Admin Unit, Customer Care and Billing Unit respectively. The back end in turns work on the customer account history after rating the status will generate the mini statement of bills and passes it through customer database and finally display the output as Mini Statement of calls (customer name, Name of town to where the call was made, Town code, per each customer).The problem with the existing system model is that the billing engine calculates only the duration of calls and the amount for each customer. But the proposed model incorporate into the mini summary report the name of customer, the name of the town to where the calls was made, the town code per each customer.

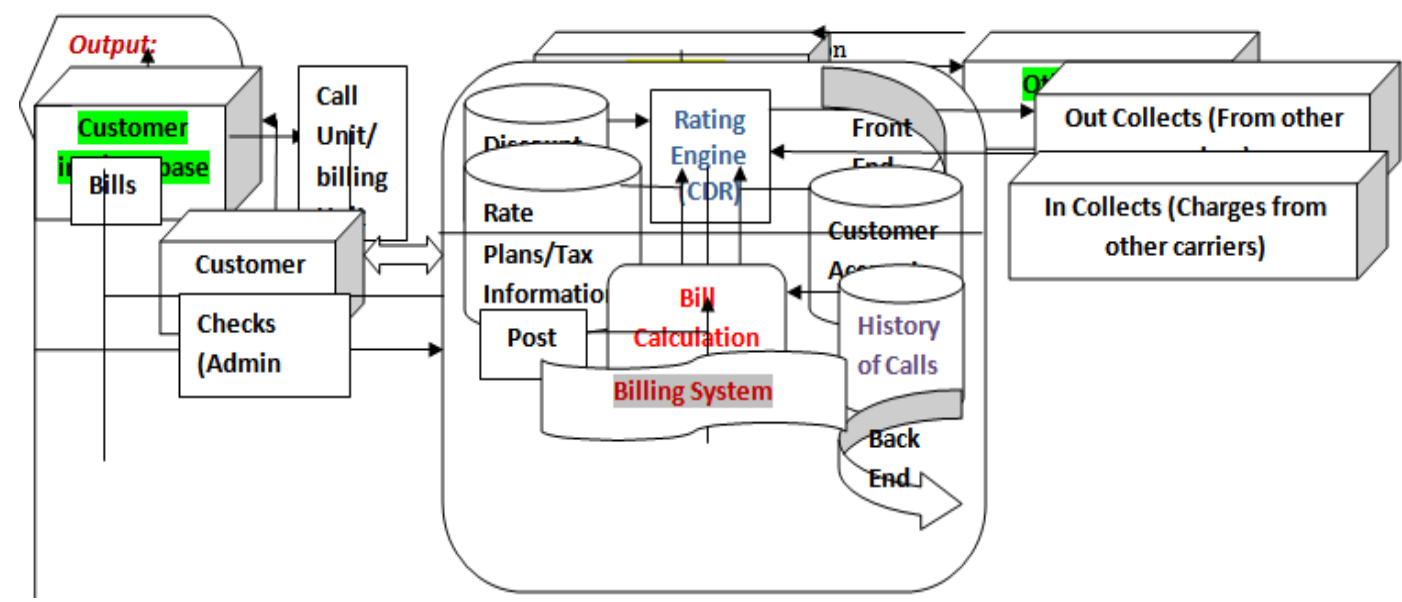

Fig. II Previous model of Billing System Architecture

Fig. II has Network centre where calls goes out from the network or comes in to the network. The usage information goes into the main billing system that has two compartments the front and the back end. The front end contains the rating engine of the billing system. This chamber has three components namely:

Discount Information that handles any bills concerning discounts charges. Rate Plans/Tax Information is aspect of the front that handles any data bundles and tax issues. The Customer Accounts is where the billing engine gets account details of customer. Also the billing engine has the back end which works simultaneously 
with front end. Calculation of bills using calls history of customer posted to it from Admin Unit, Customer Care and Billing Unit respectively. The back end in turns work on the customer account history after rating the status will generate the bill and passes it through customer database and finally display the output as call duration and Amount.

The problem with the existing system model is that the billing engine calculates only the duration of calls and the amount for each customer.

\subsection{Research Design and Sampling Procedure}

\section{Method}

A cross sectional survey design was adopted to identify the parameters existing and those not existing on the output summary of the Etisalat network. The population of study were drawn from the four Units that made up the organizational structure; Billing Unit, Customer Care Unit, Call Unit and Admin Unit.

\subsection{Instrumentation}

The uses of formal and informal interview were employed by the researcher in the collection of data from the respondents. However, average of 8 respondents was interviewed from all the four units of the organization. In each of these units 2 numbers of respondents were interviewed. Each respondent were asked 8 questions each concerning their output parameters.

\subsection{Data Collection/Analysis}

The survey was conducted through face-to-face contact with the respondents. The interview question was based on two parameters; favourable and unfavourable conditions. During the analysis; the researchers were able to get up $100 \%$ in an unfavourable condition which means that there is problem of not having customer name, town name and town code in the call summary displayed after calls. This implies that there are 3 unfavourable responses from the respondents
* Customer name are not displayed.
* Town name are not displayed
* Town code are not displayed

Hence, the computer source code program and corresponding output to solve this problem were shown below:

\subsubsection{Program Source Code}

Program CusBill(input,output,outf);

uses crt;

const $\quad$ TownCode $\mathrm{A}=24$;

TownCodeB $=24$;

TownCodeC $=24$;

TownCode $\mathrm{D}=50$;

var

custname;town: string[30];

i: integer;wrong:boolean;

ZoneCode;TownCode;more:char;outf:text;

bill:real;Duration;Amount;Rate;secs:integer;

begin

assign(outf;'excs/out.pas);

rewrite(outf;clrscr;more:='Y';

while more $=$ ' $\mathrm{Y}$ 'do

begin

writeln('*****CUSTOMER BILL****');

write('Customer Name:');

readln(custname);

write('Name of town to where call was made:');

readln(town);

write('TownCode(An alphabet):');

readln(TownCode);Towncode:=upcase(TownCode);

write('Duration of Calls (In seconds):');

readln(Duration);

write('Rate (In kobo) for TownCode A is:');

readln;

write('Rate (In kobo) for TownCode B is:'); 
readln;

write('Rate (In kobo) for TownCode C is:');

readln;

write('Rate (In kobo) for TownCode D is:');

readln;

write('Amount is Always (Duration of Calls * Rate (In kobo per second)');

Case TownCode of

'A': begin

Amount:=(Duration*Rate);

end;

'B' begin

Amount:=(Duration*Rate);

end;

'C' begin

Amount:=(Duration*Rate)

end;

'D' begin

Amount:=(Duration*Rate);

end;

else

end; (*end of Case Statement*);

if not wrong then

begin

\section{WRITELN}

if secs $>=3600$ then

bill:= bill-(0.2*bill);

writeln(outf, ${ }^{*} * * *$ CUSTOMER BILL $\left.* * * * '\right)$;

writeln(outf,'Customer Name:',Custname:1);

writeln(outf,'Name of town to where call was made:',town);

writeln(outf,'Town Code:',Towncode:1);

writeln(outf,'Duration of call:',secs:8,'seconds');

writeln(outf,'Amount Payable (in Naira):',bill:8:2);

writeln(outf); writeln(outf)e

writeln;writeln(Any more customer records?(y/n)');

readln(more);

more:=upcase(more);

end.

end; $\{$ of while $\}$ close (outf)

\subsubsection{New output the Model}

\section{$* * *$ CUSTOMER BILL}

Customer Name : UCHE OKEKE

Name of town to where call was made: ENUGU

Town Code (An alphabet): A

Duration of calls (In seconds): 59

Rate (In kobo) for TownCode A is: 24

Rate (In kobo) for TownCode B is: 24

Rate (In kobo) for TownCode C is: 24

Rate (In kobo) for TownCode D is: 50

Amount is Always (Duration of Calls*Rate (In kobo per second)

\section{$* * *$ CUSTOMER BILL****}

Customer Name: OLAJUMOKE SEFINAT

Name of town to where call was made: IJEBU ODE

Town Code (An alphabet): B

Duration of calls (In seconds): 450 
Rate (In kobo) for TownCode A is: 24

Rate (In kobo) for TownCode B is: 24

Rate (In kobo) for TownCode C is: 24

Rate (In kobo) for TownCode D is: 50

Amount is Always (Duration of Calls*Rate (In kobo per second)

***CUSTOMER BILL****

Customer Name: UMARU MOHAMMED

Name of town to where call was made: JALINGO

Town Code (An alphabet): C

Duration of calls (In seconds): 60

Rate (In kobo) for TownCode A is: 24

Rate (In kobo) for TownCode B is: 24

Rate (In kobo) for TownCode C is: 24

Rate (In kobo) for TownCode D is: 50

Amount is Always (Duration of Calls*Rate (In kobo per second)

***CUSTOMER BILL****

Customer Name: JIBRIN AHMED

Name of town to where call was made: KANO

Town Code (An alphabet): D

Duration of calls (In seconds): 80

Rate (In kobo) for TownCode A is: 24

Rate (In kobo) for TownCode B is: 24

Rate (In kobo) for TownCode C is: 24

Rate (In kobo) for TownCode D is: 50

Amount is Always (Duration of Calls*Rate(In kobo per second)

***CUSTOMER BILL****

Customer Name: EMMANUEL JAMIL

Name of town to where call was made: ABUJA

Town Code (An alphabet): D

Duration of calls (In seconds): 216

Rate (In kobo) for TownCode A is: 24

Rate (In kobo) for TownCode B is: 24

Rate (In kobo) for TownCode C is: 24

Rate (In kobo) for TownCode D is: 50

Amount is Always (Duration of Calls*Rate (In kobo per second)

***CUSTOMER BILL****

Customer Name: JAMIL MOHAMMED

Name of town to where call was made: ABEOKUTA

Town Code (An alphabet): B

Duration of calls (In seconds): 50

Rate (In kobo) for TownCode A is: 24

Rate (In kobo) for TownCode B is: 24

Rate (In kobo) for TownCode C is: 24

Rate (In kobo) for TownCode D is: 50

Amount is Always (Duration of Calls*Rate (In kobo per second)

$* * *$ CUSTOMER BILL****

Customer Name: OBINNA AFAM

Name of town to where call was made: AWKA

Town Code (An alphabet): A

Duration of calls (In seconds): 100

Rate (In kobo) for TownCode A is: 24

Rate (In kobo) for TownCode B is: 24

Rate (In kobo) for TownCode C is: 24 
Rate (In kobo) for TownCode D is: 50

Amount is Always (Duration of Calls*Rate (In kobo per second)

***CUSTOMER BILL****

Customer Name: EMEKA OKAFOR

Name of town to where call was made: UMUAHIA

Town Code (An alphabet): A

Duration of calls (In seconds): 68

Rate (In kobo) for TownCode A is: 24

Rate (In kobo) for TownCode B is: 24

Rate (In kobo) for TownCode C is: 24

Rate (In kobo) for TownCode D is: 50

Amount is Always (Duration of Calls*Rate (In kobo per second)

$* * *$ CUSTOMER BILL****

Customer Name: ISAIAH UCHE

Name of town to where call was made: SOKOTO

Town Code (An alphabet): C

Duration of calls (In seconds): 3,800

Rate (In kobo) for TownCode A is: 24

Rate (In kobo) for TownCode B is: 24

Rate (In kobo) for TownCode C is: 24

Rate (In kobo) for TownCode D is: 50

Amount is Always (Duration of Calls*Rate (In kobo per second)

***CUSTOMER BILL****

Customer Name: CHINENYE OBI

Name of town to where call was made: ABUJA

Town Code (An alphabet): $\mathrm{C}$

Duration of calls (In seconds): 340

Rate (In kobo) for TownCode A is: 24

Rate (In kobo) for TownCode B is: 24

Rate (In kobo) for TownCode C is: 24

Rate (In kobo) for TownCode D is: 50

Amount is Always (Duration of Calls*Rate (In kobo per second)

\subsubsection{Output of old Model Designed}

CUSTOMER ONE

your duration of last call was 300 Seconds

the amount is $\mathrm{N} 53.70$

CUSTOMER TWO

your duration of last call was 100 Seconds

the amount is $\mathrm{N} 20.61$

\section{CUSTOMER THREE}

your duration of last call was 3,600 Seconds

the amount is $\mathrm{N} 1007.56$

\section{CUSTOMER FOUR}

your duration of last call was 150 Seconds the amount is $\mathrm{N} 34.05$

CUSTOMER FIVE

your duration of last call was 1,800 Seconds the amount is N503.78

CUSTOMER SIX

your duration of last call was 150 Seconds the amount is $\mathrm{N} 26.85$

CUSTOMER SEVEN

your duration of last call was 450 Seconds

the amount is N125.94

CUSTOMER EIGHT 
your duration of last call was 300 Seconds the amount is N53.70

your duration of last call was 75 Seconds

CUSTOMER NINE

the amount is $\mathrm{N} 13.43$

CUSTOMER TEN

your duration of last call was 225 Seconds

the amount is N62.97

\section{Result}

The computer source code program shows that

* Customer can now have a mini statement of calls having customer name, town name, town code, duration of calls and amount.

* Security of customer calls can now be guaranteed in terms of the new addition that is customer name, town name and town code.

$\$$

\section{Conclusion}

Based on the computer program source code and output, customers can now have a mini statement of bills that contains the customer name, town name, town code, duration of calls and amount immediately after calls. The implication of this research is that it could guarantee more security in GSM calls and also help to strengthen their billing system output.

\section{Recommendations}

The Nigerian Communication Commission should, by way of policy, assume the full control and management of all the network operators such that all mobile network operators would make use of available resources to achieve customers' satisfactions in terms of billing system.

2. The network operators should reduce their expenditure on promotional activities and brand building, but rather invest on network extensions, quality service delivery and product and services outlets infrastructures, while also concentrating on bill reduction strategies as a way of increasing subscribers' base.

\section{References}

[1] A.T Bell, Telecommunications billing, A Journal on Telecom billing 2005 pg1-30.

[2] NCC Press Release, (2011). "Quarterly Summary of Telephone Subscribers in Nigeria, (internet) (http://www. ncc.gov.ng) 24 April, 2011.

[3] NCC Press Release, (2007). "Quarterly Summary of Telephone Subscribers in Nigeria, (internet) (http://www. ncc.gov.ng) 19 September, 2011.

[4] Etisalat Nigeria Plc official website http:// www.etisalatnigeria.com Quelch and Hoff, Customizing Global Marketing Harvard Business Review 2006 64, 59-68.

[5] Omotayo and Joachim, Customer Service in the Retention of Mobile Phone Users in Nigeria

[6] African Journal of Business Management 2008 Vol.2(2), pp 026-031, February 2008, ISSN 1993 - 8233.

[7] Babalola and Adegoke, Quality of Service Analysis of GSM Telephone System in Nigeria

[8] American Journal of Scientific and Industrial Research, 2011. 